
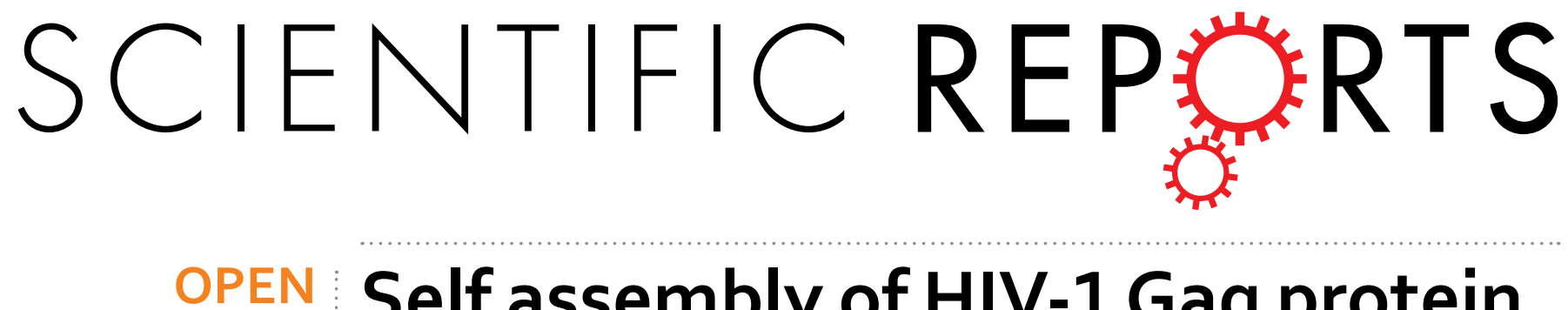

\title{
Self assembly of HIV-1 Gag protein on lipid membranes generates $\mathrm{PI}(4,5) \mathrm{P}_{2} /$ Cholesterol nanoclusters
}

Received: 18 August 2016

Accepted: 22 November 2016

Published: 23 December 2016

\author{
Naresh Yandrapalli ${ }^{1}$, Quentin Lubart ${ }^{2}$, Hanumant S. Tanwar ${ }^{3}$, Catherine Picart ${ }^{2}$, \\ Johnson Mak ${ }^{3}$, Delphine Muriaux ${ }^{1} \&$ Cyril Favard $^{1}$
}

The self-assembly of HIV-1 Gag polyprotein at the inner leaflet of the cell host plasma membrane is the key orchestrator of virus assembly. The binding between Gag and the plasma membrane is mediated by specific interaction of the Gag matrix domain and the $\mathrm{PI}(4,5) \mathrm{P}_{2}$ lipid $\left(\mathrm{PIP}_{2}\right)$. It is unknown whether this interaction could lead to local reorganization of the plasma membrane lipids. In this study, using model membranes, we examined the ability of Gag to segregate specific lipids upon self-assembly. We show for the first time that Gag self-assembly is responsible for the formation of PIP lipid nanoclusters, enriched in cholesterol but not in sphingomyelin. We also show that Gag mainly partition into liquiddisordered domains of these lipid membranes. Our work strongly suggests that, instead of targeting pre-existing plasma membrane lipid domains, Gag is more prone to generate $\mathrm{PIP}_{2} /$ Cholesterol lipid nanodomains at the inner leaflet of the plasma membrane during early events of virus assembly.

The retroviral Gag protein drives the assembly process of the Human Immunodeficiency Virus type 1(HIV-1) particles $^{1,2}$. This protein is synthesized as a polyprotein Pr55 Gag, which contains three major structural domains, namely matrix (MA), capsid (CA) and nucleocapsid (NC), as well as two spacer peptides, sp1 and sp2 and an unstructured C-terminus p6 peptide. Each of these domains are known to have a specific and distinct function during the viral assembly process. Importantly, the N-terminal MA domain targets Gag to the plasma membrane and mediates membrane binding, the CA domain is responsible for Gag-Gag interaction and self-assembly and the NC domain recruits the viral RNA that also acts as a scaffold for viral particle assembly ${ }^{3}$. Although it has recently been shown that HIV assembly may be initiated in the cytosol ${ }^{4}$, it is commonly accepted that the formation of large HIV-1 assembly complex mainly occurs at the plasma membrane (PM) of the virus producing cells. Two main features of the N-terminal MA domain of Gag govern the HIV-1 Gag inner leaflet PM binding: the N-terminal myristate and the highly basic region (HBR) that contains a specific binding pocket for the phosphatidyl inositol $(4,5)$ bisphosphate lipid $\left(\mathrm{PI}(4,5) \mathrm{P}_{2} \text { or } \mathrm{PIP}_{2}\right)^{5}$. $\mathrm{PIP}_{2}$ has been shown to play a major role in HIV-1 assembly in cells since cellular depletion of $\mathrm{PIP}_{2}$ decrease the efficiency of viral particle release ${ }^{6}$. In addition, PIP $_{2}$ is also enriched in the virus envelope relative to the PM of the virus-producing cells ${ }^{7}$. This lipidomic analysis also shows that HIV particles are enriched in sphingolipids and cholesterol $^{7}$ supporting the idea that the viral particles are released from the so called "rafts" domains. Historically, Gag has been shown to associate with detergent resistant membrane (DRM) by cell membrane fraction assays ${ }^{8,9}$. Nevertheless, the exact composition of these "rafts" or DRM is controversial ${ }^{10,11}$. Indeed, many different types of "rafts" may exist within the plasma membrane as long as they are enriched in cholesterol and sphingolipids ${ }^{12}$. However, Gag self-assembly occurs at the inner leaflet of the cellular PM where sphingolipids are poorly present. Moreover, natural $\mathrm{PIP}_{2}$, the main interacting partner of Gag, has a polyunsaturated fatty acyl residue at the sn-2 position of the glycerol backbone. This acyl chain polyunsaturation is not in favour of $\mathrm{PIP}_{2}$ partitioning into rafts ${ }^{13}$. To circumvent such a constrain, Saad et al. suggested a model based on NMR data using truncated acyl chains $\mathrm{PIP}_{2}{ }^{5}$. In this model, the sn-2 acyl chain of the $\mathrm{PIP}_{2}$ is removed from the plane of the membrane and trapped into an hydrophobic pocket of MA. However, coarse grained dynamics studies ${ }^{14}$ and new NMR experiments using full length acyl chain PIP $_{2}{ }^{15}$ showed the opposite. Finally, recent experiments using either a multimerizable matrix domain of HIV-1 Gag ${ }^{16}$ or RSV Gag $^{17}$

${ }^{1}$ CNRS-Université Montpellier, Centre d'études d'agents Pathogenes et de Biotechnologies pour la Santé, Montpellier, France. ${ }^{2}$ CNRS-Université Grenoble, Institut National de Physique, Grenoble, France. ${ }^{3}$ Deakin University, School of Medicine, Melbourne, Australia. Correspondence and requests for materials should be addressed to D.M. (email: delphine.muriaux@cpbs.cnrs.fr) or C.F. (email: cyril.favard@cpbs.cnrs.fr) 


\begin{tabular}{|c|c|c|c|c|c|c|c|c|}
\hline & \multicolumn{2}{|c|}{ Basic } & \multicolumn{2}{|c|}{ Substituted Basic } & \multicolumn{2}{|c|}{ Inner Leaflet $^{*}$} & \multicolumn{2}{|c|}{ Rafts-mimicking ${ }^{\#}$} \\
\hline & Lipid & Mol\% & Lipid & Mol\% & Lipid & Mol\% & Lipid & Mol\% \\
\hline & EggPC & 68 & Egg PC & 66 & Egg PC & 17 & DOPC & 31 \\
\hline & Brain PS & 30 & Brain PS & 30 & Brain PS & 17 & DOPS & 6 \\
\hline & & & Brain $\mathrm{PI}(4,5) \mathrm{P}_{2}$ & 2 & Brain SM & 9 & Brain SM & 30 \\
\hline & & & & & Cholesterol & 30 & Cholesterol & 25 \\
\hline & & & & & Liver PE & 25 & GM1 & 1 \\
\hline \multirow{2}{*}{ LUV } & Brain $\mathrm{PI}(4,5) \mathrm{P}_{2}$ & 0 or 1.5 & & & Brain $\mathrm{PI}(4,5) \mathrm{P}_{2}$ & 0 & Brain $\mathrm{PI}(4,5) \mathrm{P}_{2}$ & 5 \\
\hline & TF-PIP ${ }_{2}$ & 2 or 0.5 & & & TF-PIP $_{2}$ & 2 & TF-PIP ${ }_{2}$ & 2 \\
\hline \multirow{2}{*}{ SLB } & Brain $\mathrm{PI}(4,5) \mathrm{P}_{2}$ & 1 & Brain SM or Chol. & 1 & Brain $\mathrm{PI}(4,5) \mathrm{P}_{2}$ & 1 & Brain $\mathrm{PI}(4,5) \mathrm{P}_{2}$ & 6 \\
\hline & TF-PIP ${ }_{2}$ & 1 & TF-SM or TF-Chol & 1 & TF-PIP ${ }_{2}$ & 1 & TF-PIP ${ }_{2}$ & 1 \\
\hline \multirow{2}{*}{ GUV } & Brain $\mathrm{PI}(4,5) \mathrm{P}_{2}$ & 1 & & & Brain $\mathrm{PI}(4,5) \mathrm{P}_{2}$ & 1 & Brain $\mathrm{PI}(4,5) \mathrm{P}_{2}$ & 6 \\
\hline & TF-PIP ${ }_{2}$ & 1 & & & TF-PIP ${ }_{2}$ & 1 & TF-PIP $_{2}$ & 1 \\
\hline
\end{tabular}

Table 1. Different lipid composition of LUV, SLB and GUV used in the single labeled lipid experiments.

\#Adapted from ref. 28. "Adapted from ref. 16.

also exhibited contradictory results regarding their partitioning in lipid domains of giant unilamellar vesicles (GUVs).

Therefore, the ability of the Gag/PIP ${ }_{2}$ complex to partition preferentially into "raft" domains or more generally into PM pre-existing domains to enhance virus assembly is still a matter of controversy ${ }^{18}$. In order to elucidate whether Gag would bind to pre-existing "rafts" or lipid domains in the PM or generate its own lipid domains for assembling, we first decided to monitor its binding and partitioning to single or dual-phase model membranes made with simple and complex lipid compositions (see Table 1 for detailed composition of lipid mixtures). On GUVs, we observed that Gag was mainly partitioning into liquid-disordered $\left(L_{d}\right)$ phase, i.e. more likely out of "rafts" microdomains in the dual-phase model membrane and that Gag was not generating micrometer range lipid phase separation in single phase model membranes.

We therefore monitored, at the nanoscale level, a possible $\mathrm{PIP}_{2}$, cholesterol (Chol) and sphingomyelin (SPM) reorganization during Gag self-assembly. Unfortunately, direct imaging of lipid nanodomains generation is not an easy task. Even the presence of pre-existing lipid nanodomains has indirectly been revealed by monitoring lipid diffusion ${ }^{19-23}$. Although accurate in detecting and measuring the size of pre-existing nanodomains, direct imaging is not fast enough to follow the generation of nanodomains. Therefore, we decided to use indirect approaches such as fluorescence quenching ${ }^{24,25}$ or Forster Resonant Energy Transfer (FRET) ${ }^{26}$. FRET or self-quenching occurs when two (similar or different) fluorescent molecules are found together within a 3 to $10 \mathrm{~nm}$ radius.

Starting from a simple lipid mixture and using self-quenching experiments of respectively $\mathrm{PIP}_{2}$, Chol and SPM, we monitored the generation of Gag induced lipid nanodomains. We then performed the same type of experiments with dual labelling of the lipids $\left(\mathrm{Chol}\right.$ and $\mathrm{PIP}_{2}$, or SPM and $\left.\mathrm{PIP}_{2}\right)$ followed up by FRET experiments to analyse how Gag was differently sorting these lipids during its self-assembly. We finally extended this study to more complex lipid compositions mimicking either lipid "rafts" or inner leaflet of the PM.

Our results show that Gag self-assembly is able to generate $\mathrm{PIP}_{2}$ nanodomains on model membranes with any of the composition we tested here. Importantly, these nanodomains contain Chol but not SPM.

\section{Results}

Since PIP ${ }_{2}$, Chol and SPM have been shown to be enriched in the virus envelope ${ }^{7}$, the impact of Gag self-assembly on their lateral distribution was tested in different types of model membranes (Fig. 1A) exhibiting different lipid compositions. Large unilamellar vesicles (LUVs) were firstly used in order to easily control the protein over lipid molecular ratio. Given that the plasma membrane of HIV producing cells ( $\sim 10 \mu \mathrm{m}$ in diameter) has reduced level of curvature compared to these LUVs ( $100 \mathrm{~nm}$ in diameter), we have also used supported lipid bilayers (SLBs) as membrane models for analyses. Gag is known to bind specifically $\mathrm{PIP}_{2}{ }^{27}$. We then started with a simple $\mathrm{PIP}_{2}$ containing lipid mixture (PC/PS/PIP 2 hereafter called "basic") in which we introduced Chol or SPM (hereafter called "substituted basic"). Obviously, cellular PM have more complex lipid compositions. We therefore decided to extend our study to compositions mimicking either the inner leaflet cellular plasma membrane ${ }^{28}$ (hereafter called "inner leaflet") or the "lipid rafts" domains (i.e. two separated phases, a liquid disordered $\left(\mathrm{L}_{d}\right)$ and a liquid ordered $\left(\mathrm{L}_{o}\right)$ enriched in cholesterol and sphingomyelin, hereafter called "rafts-mimicking"16). Table 1 gives the exact compositions of the lipid mixtures used in this study. To assess a potential reorganisation of these lipids during Gag self-assembly, we used full-length Gag (FL-Gag) and different mutants. Theses mutants include: (1) a mutant of CA involved in CA-CA interaction and Gag self-assembly (WM $)^{29}(2)$ Gag lacking the C-terminus NC-sp2-p6 domains that is involved in NC-RNA association (P39). The membrane binding domain alone of Gag (MA) was also tested (see Fig. 1B). Finally, a cellular $\mathrm{PM} \mathrm{PIP}_{2}$ binding protein, the $\mathrm{PH}$ domain of EFA6 (PH-EFA6) ${ }^{30}$ and a peptide, MARCKS (151-175) that is known to laterally redistribute $\mathrm{PIP}_{2}{ }^{24}$ were used as controls.

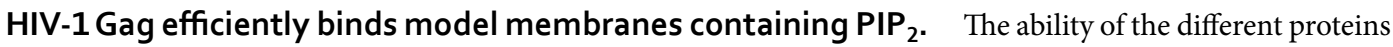
and peptide to bind LUVs and SLBs was analysed using membranes of "basic" composition. For this purpose, we monitored protein binding with two different techniques. On one hand, we performed LUV cosedimentation assays at fixed protein concentration $(1 \mu \mathrm{M})$ and increasing $\mathrm{PIP}_{2}$ concentrations. After 15 min of incubation, LUV bound proteins were separated from unbound proteins by ultra-centrifugation. The pellet $(\mathrm{P})$ and the 


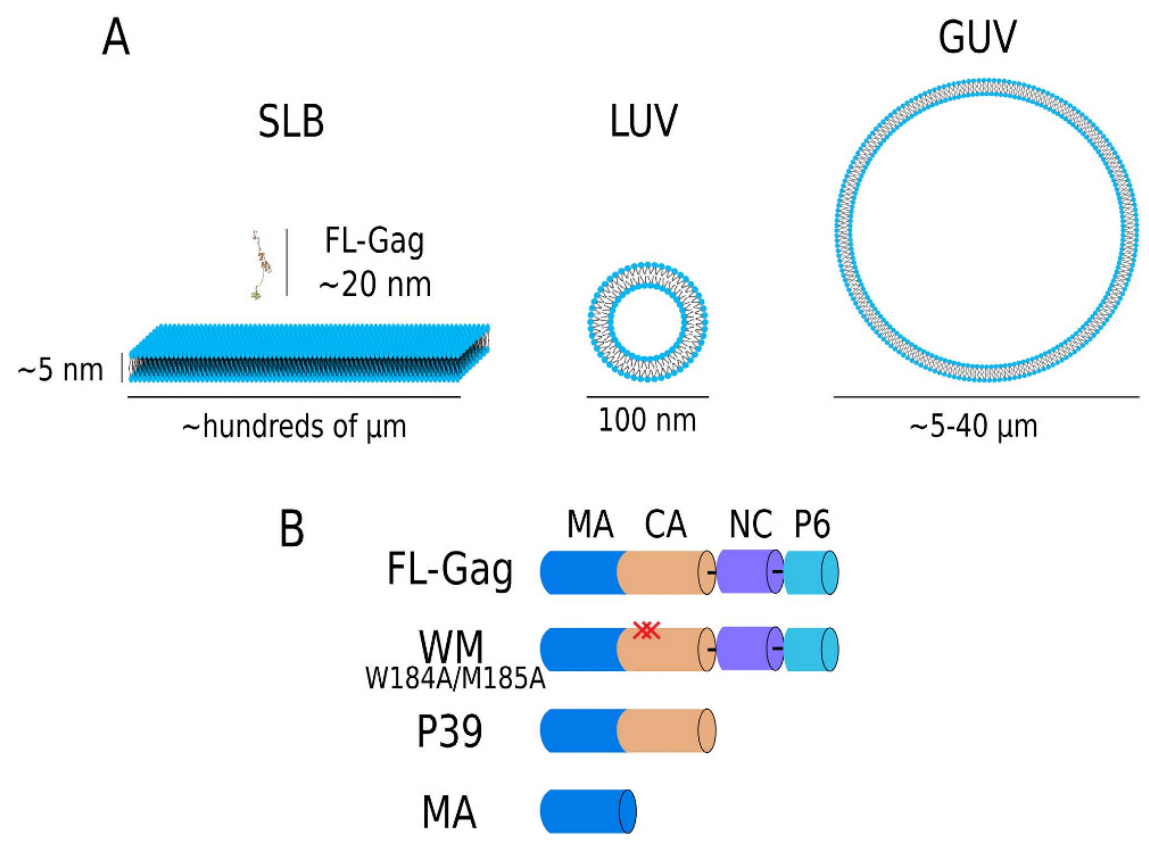

Figure 1. Scheme of the different lipid membranes and proteins used in this study. (A) Representation of the model lipid membranes and of an elongated FL-Gag protein used in this study (see Table 1 and SI Table S1 for all the different lipid compositions used in this study). (B) Schematic representation of the different Gag mutants used in this study, emphasizing the mutations and the differences in the domains present for each mutant.

supeRNAtant $(\mathrm{S})$ were deposited on a denaturating gel to quantify the amount of bound $(\mathrm{P})$ and unbound (S) proteins (Fig. 2A). On the other hand, we used Quartz Crystal Microbalance (QCM) to monitor the association of proteins to SLBs containing a fixed $\mathrm{PIP}_{2}$ concentration. SLBs were formed onto the quartz crystal. Proteins were then injected at increasing concentrations (from $10^{-2}$ to $10 \mu \mathrm{M}$ ) and the change in the resonance frequency of the quartz crystal was monitored (Fig. 2B). This change in frequency is directly connected to the mass increase at the surface of the quartz crystal.

The ratio of membrane bound to unbound protein is linked to an apparent partition coefficients $\left(K_{p}\right) . K_{p}$ were determined by fitting, with eq. 1 , the plots of membrane bound protein with either increasing concentrations of lipids (Fig. 2C) or increasing concentration of protein (Fig. 2D). Except for MA, $K_{p}$ obtained for other proteins were less than $1 \mu M$. The results, summarized in Table 2, are in good agreement with the previously obtained $K_{p}$ values for some of the different proteins used in this study. Interestingly, the $K_{p}$ values of MARCKS and PH-EFA6 $\left(0.7<K_{p}<0.8 \mu M\right)$ were found to be in the range of the one obtained for Gag (FL) and its mutants $\left(0.2<K_{p}<0.8 \mu M\right)$. This shows that the ratio of membrane bound over total protein will be equivalent for all these proteins, allowing, therefore, direct comparisons of their respective role on the lateral sorting of $\mathrm{PIP}_{2}$ after membrane binding.

FL-Gag is mainly partitioning to $\mathrm{L}_{d}$ phases and does not induce micrometer phase separation. We firstly monitored the possible existence of Gag induced micrometer-range $\mathrm{PIP}_{2}$ domains by imaging the surface distribution of a fluorescently labelled PIP $_{2}$ (namely Top-Fluor PIP $_{2}$ or TF-PIP ${ }_{2}$ ) and a fluorescently labeled FL-Gag (FL-Gag Alexa 549 or A549-FL-Gag) in "basic" GUVs. As expected, both TF-PIP 2 and A549-FL-Gag showed a uniform distribution over the surface of the GUV (Fig. 3A). The same uniform distribution was also observed with "inner leaflet" GUVs (Fig. 3A). Spatial auto-correlation of the fluorescence of either PIP ${ }_{2}$ or FL-Gag (Fig. 3B) both exhibited fast decorrelation showing that the fluorescent labelling of $\mathrm{PIP}_{2}$ is not inducing micrometer partitioning per se and that, if they exist, the PIP clusters are less than $200 \mathrm{~nm}$ size (i.e. below the diffraction limit). Interestingly, labelled FL-Gag was essentially restricted to $\mathrm{L}_{d}$ phases in the "rafts-mimicking" GUVs (Fig. 3C and D), confirming what was already observed by Keller et al. ${ }^{16}$ with a multimerized derivative of MA. This strongly suggested that FL-Gag preferentially binds and remains in non "rafts"/ $\mathrm{L}_{o}$ lipid phases.

HIV-1 Gag self-assembly induces $\mathrm{PIP}_{2}$ nanoclustering. The lack of micrometer scale $\mathrm{PIP}_{2}$ induced domains lead us to monitor, using self-quenching experiments, a possible $\mathrm{PIP}_{2}$ lateral redistribution upon Gag self-assemby on LUVs or SLBs. As stated in the introduction, self-quenching occurs when two fluorescent molecules are close to each other $(3<\mathrm{r}<10 \mathrm{~nm})$. To perform these experiments, we first controlled that increasing concentrations of MARCKS induced an increasing quenching of the TF-PIP ${ }_{2}$ as it was reported before with

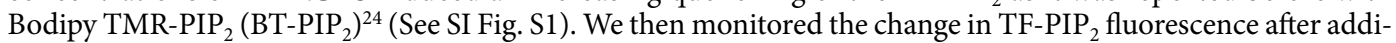
tion of Gag or its mutants on "basic" LUVs in order to correctly control the protein to accessible PIP $_{2}$ ratio 
A

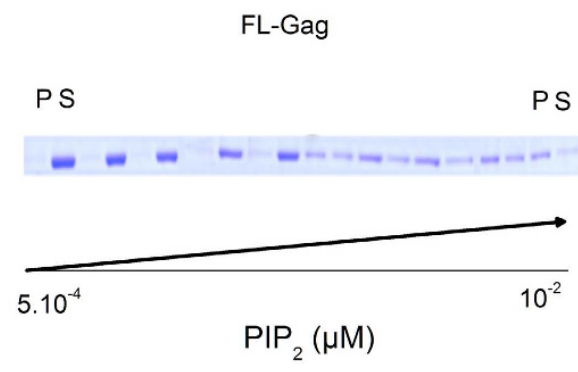

C

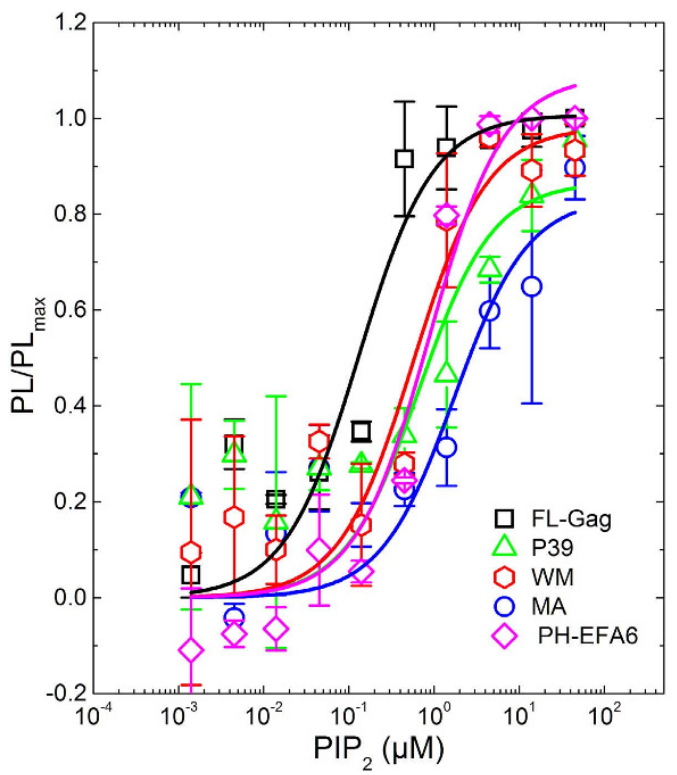

PS
B

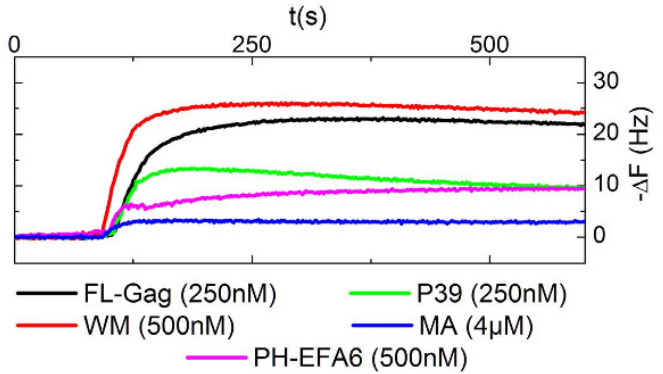

D

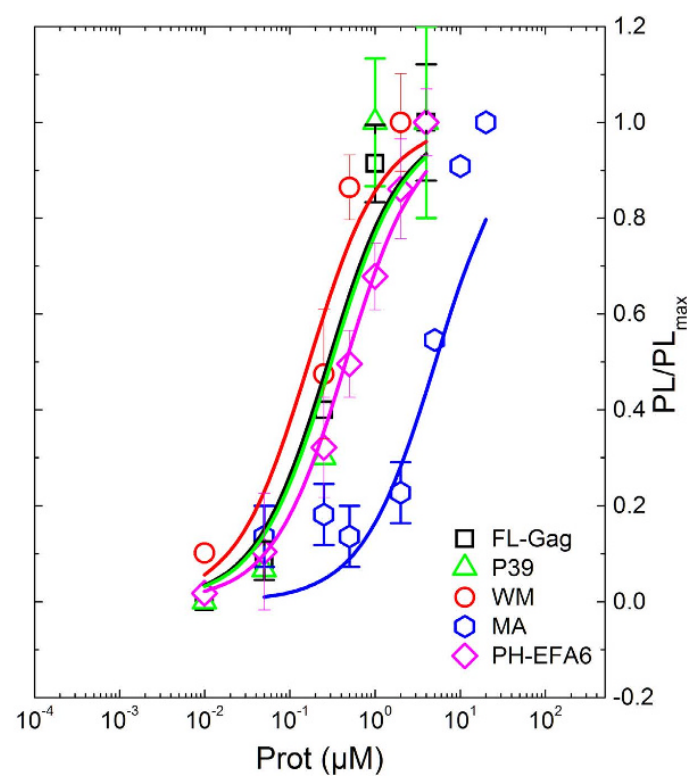

Figure 2. Binding of FL-Gag, its mutants and PH-EFA6 to "basic" lipid membranes. (A) Typical SDSPAGE obtained after co-sedimentation assay for FL-Gag with increasing concentrations of LUV containing $2 \%$ mol PIP $_{2}$ (P:Pellet, S:SupeRNAtant). (B) Typical change in resonance frequency observed during QCM-D experiments after addition of the different proteins at different concentrations on a basic composition SLB. $(\mathbf{C}$ and $\mathbf{D})$ Binding isotherm curve obtained from co-sedimentation assays $((\mathbf{C}), \mathrm{n}=3)$ and QCM-D experiments $((\mathbf{D}), \mathrm{n}=2)$ for the different proteins used in this study. Experimental values were fitted using equation $1 . K_{p}$ obtained from these binding isotherms are summarized in Table 2.

\begin{tabular}{|c|c|c|c|c|c|}
\hline \multirow[b]{2}{*}{ Protein } & $K_{p}(\mu M)$ & $K_{p}(\mu M)$ & \multirow[b]{2}{*}{$K_{p}(\mu M)$} & \multirow[b]{2}{*}{ Lip. Comp (mol \%) } & \multirow[b]{2}{*}{ Ref } \\
\hline & LUV & SLB & & & \\
\hline $\mathrm{FL}$ & $0.13 \pm 0.05$ & $0.40 \pm 0.11$ & $\begin{array}{l}0.88 \pm 0.20 \\
\quad(\mathrm{LUV})\end{array}$ & POPS (100) & 55 \\
\hline P39 & $0.66 \pm 0.44$ & $0.47 \pm 0.26$ & & & \\
\hline WM & $0.56 \pm 0.26$ & $0.26 \pm 0.13$ & & & \\
\hline \multirow{2}{*}{ MA } & $1.72 \pm 1.05$ & & $2.46 \pm 1(\mathrm{LUV})$ & POPS (100) & 55 \\
\hline & & $7.16 \pm 3.33$ & $8.2 \pm 0.7$ (SLB) & DOPC:DOPS:PI(4,5) $\mathrm{P}_{2}(80: 15: 5)$ & 39 \\
\hline \multirow{2}{*}{ PH-EFA6 } & \multirow{2}{*}{$0.88 \pm 0.27$} & \multirow{2}{*}{$0.68 \pm 0.03$} & \multirow{2}{*}{0.5 (SLB) } & PC:PE:PS:PI(4,5) $\mathrm{P}_{2}$ & \multirow{2}{*}{30} \\
\hline & & & & $(34: 34: 30: 2)$ & \\
\hline \multirow{2}{*}{ MARCKS } & \multirow{2}{*}{ n.d. ${ }^{*}$} & \multirow{2}{*}{$0.65 \pm 0.23$} & \multirow{2}{*}{$4.4 \pm 1$ (LUV) } & PC:PI $(4,5) \mathrm{P}_{2}$ & \multirow{2}{*}{56} \\
\hline & & & & $(99: 1)$ & \\
\hline
\end{tabular}

Table 2. Apparent $K_{p}$ of the different proteins used in this study. "Could not be determined by this method.

$\left(P / P I P_{2}^{a c c}\right)$. We observed an increase in TF-PIP ${ }_{2}$ fluorescence (Fig. 4A) opposite to MARCKS. From Fig. $4 \mathrm{~B}$ it was evidenced that addition of increasing concentrations of FL-Gag, P39 and WM induced an increasing fluorescence 
A

"Basic"

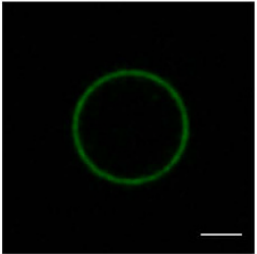

TF-PIP

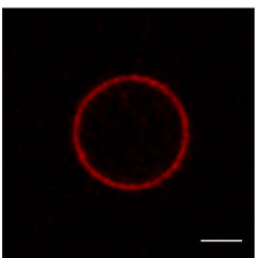

A549-FL-Gag
"Inner Leaflet"
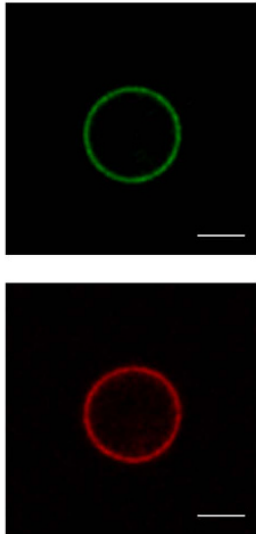

B

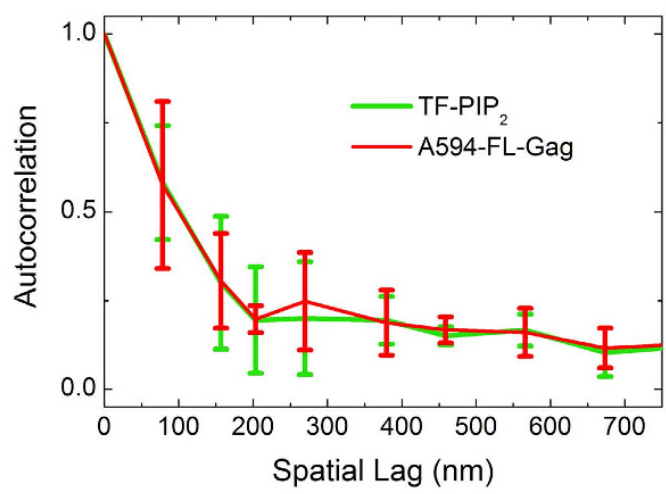

TF-PIP

A549-FL-Gag

A647-CTxB
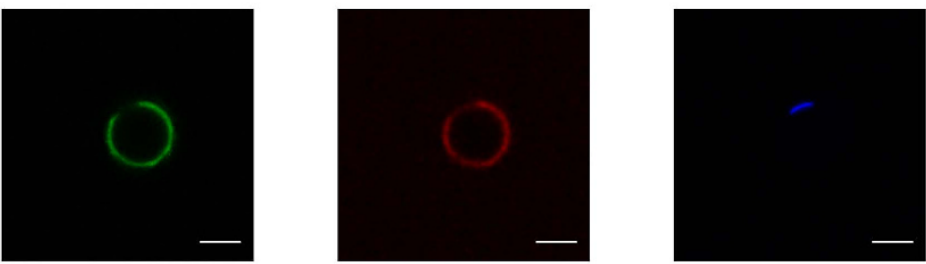

D

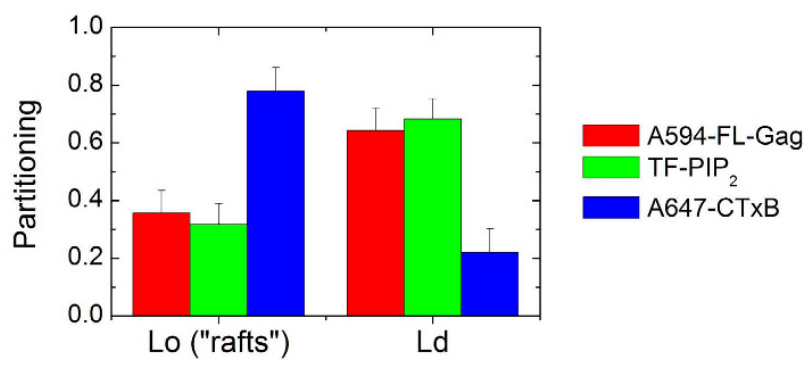

Figure 3. Fluorescence distribution of TF-PIP 2 and A594-FL-Gag on "Basic", "Inner Leaflet" and "raftsmimicking" GUVs. (A) Left, localization of TF-PIP ${ }_{2}$ (green) and A594-FL-Gag (red) in a "Basic" composition GUV. Right, localization of TF-PIP 2 (green) and A594-FL-Gag (red) in an "Inner Leaflet" composition GUV, (scale bar $5 \mu \mathrm{m}$ ). (B) Spatial autocorrelation of the fluorescence intensities of TF-PIP 2 (in green) and A594-FLGag(in red) for the "Basic" composition calculated using eq. 2, (mean \pm s.d., $n=4)$. (C) Localization in "Raft" GUVs (F) of TF-PIP (green), A594-FL-Gag (red) and GM1, a raft partitioning ganglioside, labeled with alexa 647 cholera toxin B (A647 Ctx-B)(blue), (scale bar $5 \mu \mathrm{m}$ ). (D) Partitioning in $L_{o}$ and $L_{d}$ phase of "raft" GUVs for A-594 FL, TF-PIP 2 and A647-CtxB (mean \pm s.d., $n=25)$ as determined using eq. 3.

unquenching of TF-PIP 2 . On the other hand, adding increasing concentrations of MA did not generate any change in the fluorescence of TF-PIP ${ }_{2}$. Identically, PH-EFA6, known to specifically bind PIP $_{2}$ without 
A

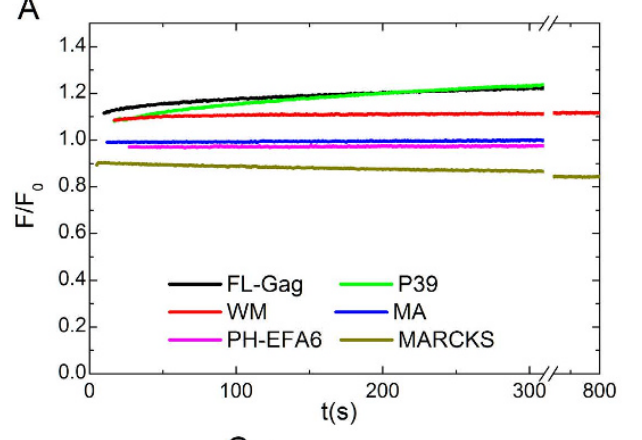

B

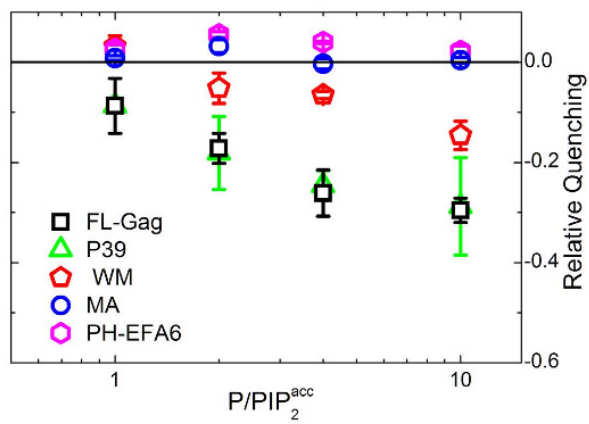

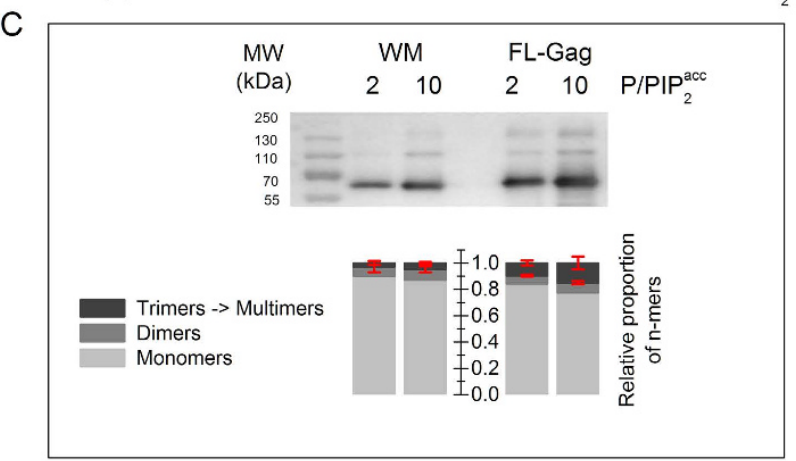

D

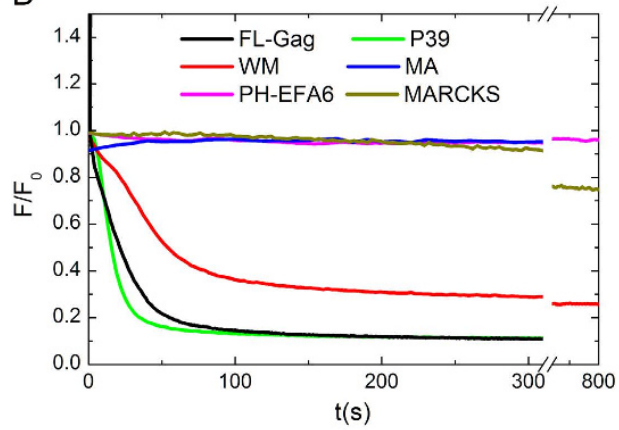

$\mathrm{F}$

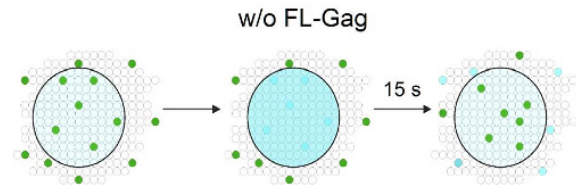

w/ FL-Gag

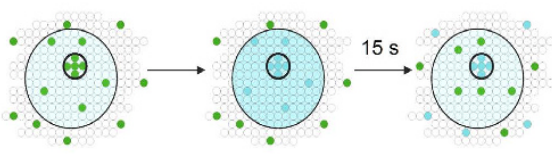

$\mathrm{E}$

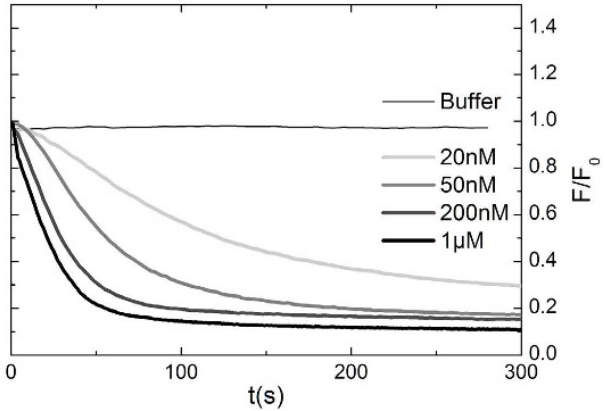

G

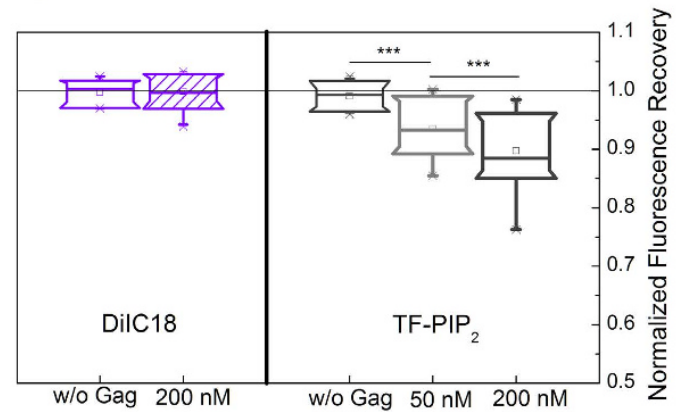

Figure 4. PIP ${ }_{2}$ nanoclustering induced by Gag self-assembly on basic composition lipid membranes. (A) Typical time course of TF-PIP ${ }_{2}$ fluorescence on LUVs after addition of the different proteins or peptide at a $\mathrm{P} / \mathrm{PIP}{ }_{2}^{a c c}=10$. (B) Relative quenching values observed for FL-Gag, its mutant, MA and PH-EFA6 on LUVs (mean \pm s.d. values of $n \geq 3$ for each P/PIP acc conditions, except P39 $(2 \leq n \leq 3)$ ). (C) Differences in WM and FL-Gag self-assembly efficiency on LUV at two different $\mathrm{P} / \mathrm{PIP}_{2}^{a c c}$ ratio. Bar graph represents the mean signal observed for monomers dimers and trimers \& multimers obtained from independent experiments (mean \pm s.d., $\mathrm{n}=3$ ). (D) Typical time course of TF-PIP ${ }_{2}$ fluorescence on SLBs after addition of $1 \mu \mathrm{M}$ of the different proteins or peptide. (E) Fluorescence time course of TF-PIP ${ }_{2}$ after addition of increasing FL-Gag concentrations. (F) Schematic representation of the effect of an immobile fraction on the fractional recovery. Upper part, without Gag, fractional recovery $=1$. Lower part, with Gag, self assembled Gag trapped $\mathrm{PIP}_{2}$ are still in the bleached area leading to a normalized fractional recovery $<1$. (G) Plot box of the fractional recoveries obtained from FRAP measurements before and after addition of increasing concentrations of FL-Gag on SLB containing either DiIC18 as a lipid analogue control (left) or TF-PIP 2 (right). (Boxes are $25,75 \%$ with bars max and min values of $\mathrm{n} \geq 15,{ }^{* *} \mathrm{p} \leq 10^{-3}$ for Student $\mathrm{t}$-test at 0.01 confidence level). 
reorganizing its lateral distribution, did not produce any effect either (Fig. 4A and B). A major difference between the Gag matured protein MA and the precursor proteins (FL-Gag, P39 and WM) is their capacity to form large oligomer complexes via the CA-CA interactions ${ }^{31}$, although WM is less efficient (two orders of magnitudes in solution) in supporting the formation of large Gag lattice, due to its inability to dimerize with nearby CA hex$\operatorname{amer}^{29}$. Interestingly, this fluorescence unquenching was always less efficient in the case of WM compared to FL-Gag (from 3 to 5 times), indicating that this fluorescence variation depended on the capacity of Gag to multimerize. To support this hypothesis, FL-Gag and WM were incubated with "basic" LUVs for 15 minutes followed by ultracentrifugation. The self-assembly states of WM and FL-Gag bound to LUVs were analysed by performing a non denaturating gel electrophoresis to preserve existing multimers, followed by immunoblot. Figure 4C showed that, in the two $P / P I P_{2}^{a c c}$ ratio tested, WM was at least two times less efficient than FL-Gag in multimerizing on membranes (Fig. 4B). This ratio was in the range of the relative efficiency of TF-PIP 2 unquenching observed between FL-Gag and WM. This confirmed the role of Gag self-assembly in the observed TF-PIP ${ }_{2}$ fluorescence changes. Although LUVs provide a simple, reliable and easy to control (in terms of protein to lipid ratios) model membranes, Gag self-assembly usually occurs on membranes with higher radius of curvature. Therefore, time course fluorescence self-quenching of TF-PIP ${ }_{2}$ were also conducted on "basic" SLBs. For FL-Gag and P39 the TF-PIP 2 fluorescence intensity of SLBs decreased (Fig. 4D). This change in intensity was opposite to the one observed with LUVs (Fig. 4A) while still being a function of the protein concentration (Fig. 4E for FL-Gag). As it was the case for LUVs, the TF-PIP 2 quenching induced by addition of the WM mutant was less efficient than in the case of FL-Gag. PH-EFA6 and MA did not induced changes in TF-PIP 2 fluorescence (Fig. 4D). Interestingly, Fig. $4 \mathrm{D}$ shows that MARCKS addition induced the same TF-PIP2 quenching on SLBs and LUVs (see Fig. 4A). This suggest that model membrane curvature (curved LUV vs flat SLBs) is responsible for the opposite quenching observed with Gag and its mutants on SLBs and LUVs (see SI Fig. S2 for detailed explanation). Altogether these results show that the self-assembly of HIV-1 Gag is generating TF-PIP ${ }_{2}$ clusters.

Diffusion of Gag is expected to slow down upon self-assembly. Hendrix et al. ${ }^{4}$ already observed that Gag multimers exhibited a diffusion coefficient(D) of $0.01 \mu \mathrm{m}^{2} . \mathrm{s}^{-1}$ at maximum. If $\mathrm{PIP}_{2} \mathrm{~s}$ are tightly trapped in Gag multimers they will diffuse with similar coefficient as that of these Gag multimers. Consequently, the fluorescence of a $1 \mu \mathrm{m}$ radius area containing these multimers and labelled $\mathrm{PIP}_{2}$, should not totally return to its initial level $15 \mathrm{~s}$ after photobleaching (Fig. 4F). This will lead the normalized fluorescence recovery value (NFR, defined in eq. 4 , $0<N F R<1)$ to be less than 1 . We monitored this change in normalized fluorescence recovery in the absence or presence of FL-Gag. For that purpose, in the time course of quenching experiments, we performed Fluorescence Recovery After Photobleaching (FRAP) experiments before (start of the quenching experiment) and after (end of the quenching experiment) addition of FL-Gag on SLBs. In the absence of FL-Gag, normalized fluorescence recovered to 1 , as expected for freely diffusing lipids (Fig. 4G, right). On the opposite, addition of increasing FL-Gag concentrations induced a decrease in $\mathrm{PIP}_{2}$ normalized fluorescence recovery as well as an increase in fluorescence quenching (previously shown in Fig. 4E). We controlled that the decrease in normalized fluorescence recovery was specific of $\mathrm{PIP}_{2}$. Indeed, we did not observe any change in the normalized fluorescence recovery of a lipid analogue fluorescent dye (diIC18, see materials in SI) in the presence or in the absence of Gag (Fig. 4G, left).

Our data clearly show that HIV-1 Gag is sorting $\mathrm{PIP}_{2}$ in the lipid membrane and that Gag self-assembly generates $\mathrm{PIP}_{2}$ nanoclusters in model membranes.

Cholesterol but not sphingomyelin is sensitive to HIV-1 Gag self-assembly. Since Chol and SPM are supposed to be the main lipid components of "raft" domains, their enrichment into the Gag self-assembly induced $\mathrm{PIP}_{2}$ nanoclusters was also assessed. $2 \% \mathrm{~mol}$ of egg phosphatidyl choline (EPC) present in our "basic" lipid composition, were substituted either by SPM or by Chol with half of them being labelled with TF derivatives (see Table 1 for detailed composition). This substitution allowed the net surface charge and the $\mathrm{PIP}_{2}$ content to be maintained, limiting any drastic change in the partitioning constant $K_{p}$. Time course fluorescence change of either TF-SPM or TF-Chol were then monitored after FL-Gag addition. Figure 5A shows that FL-Gag addition had no effect on TF-SPM fluorescence whereas Fig. 5B shows that increasing concentrations of FL-Gag induced increasing quenching of TF-Chol, as it was the case for TF-PIP 2 (Fig. 4E). This shows that FL-Gag self- assembly is able to generate Chol-enriched lipid nanodomains whereas it is not changing the SPM lateral distribution. Co-clustering of these molecules was also assessed by simultaneously monitoring the change in fluorescence upon addition of

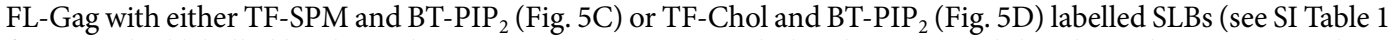
for exact dual labelled lipid membranes compositions). TF-Chol and BT-PIP 2 exhibited simultaneous quenching after addition of various concentration of FL-Gag, suggesting that TF-Chol and BT-PIP 2 are co-clustered in Gag self-assembly induced nanodomains. In contrast, while BT-PIP 2 was quenched as previously, TF-SPM exhibited a surprising apparent fluorescence unquenching after Gag addition. This was an unexpected result regarding the TF-SPM quenching induced by FL-Gag self-assembly in the presence of unlabelled PIP $_{2}$ (Fig. 5A). Since this apparent unquenching of TF-SPM upon addition of FL-Gag only occurred when BT-PIP 2 was present (see Fig. 5A vs Fig. 5C), we checked if TF-SPM (as a donor) and BT-PIP 2 (as an acceptor) were able to exhibit FRET. Donor FRET efficiency has been shown to be a function of acceptor concentration in a lipid bilayer ${ }^{32}$. This means that the FRET efficiency will decrease if acceptor $\left(\mathrm{BT}-\mathrm{PIP}_{2}\right)$ local concentration decreases and that, as a consequence, the fluorescence of the donor (TF-SPM) will increase. We tested this by performing acceptor photobleaching experiments in order to decrease the acceptor concentration, locally and reversibly. We observed that, in the area where the acceptor $\left(\mathrm{BT}_{-\mathrm{PIP}}\right.$ ) was bleached, the fluorescence of the donor (TF-SPM) increased. This effect was abolished when concentrations were re-equilibrated by diffusion (see SI Fig. 3 ). In our TF-SPM/BT-PIP ${ }_{2}$ co-clustering assay, we observed a decrease in BT-PIP 2 due to Gag self-assembly induced quenching and concomitantly, a non reversible TF-SPM fluorescence increase due to loss in FRET efficiency induced by decreasing concentration of acceptor (TF-PIP 2 ). This can only be explained by considering that Gag self-assembly clusters BT-PIP ${ }_{2}$ without TF-SPM. 
A

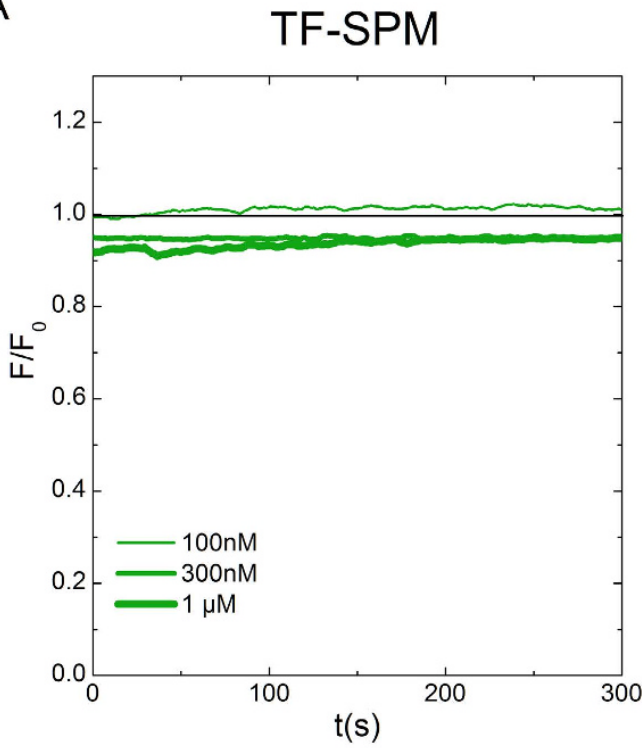

C

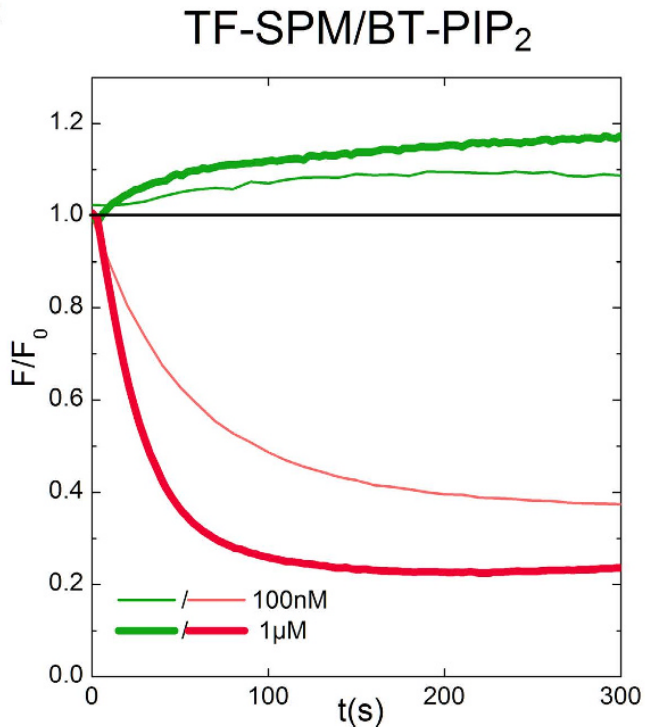

B

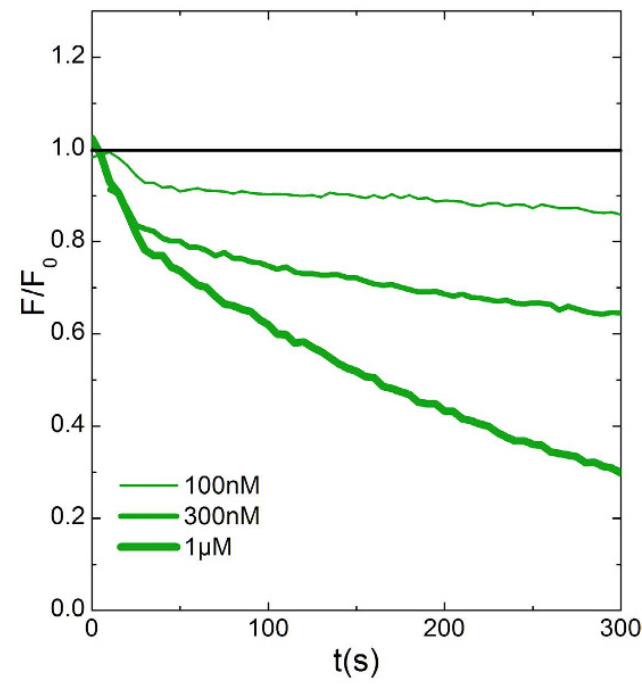

D

TF-Chol/BT-PIP 2

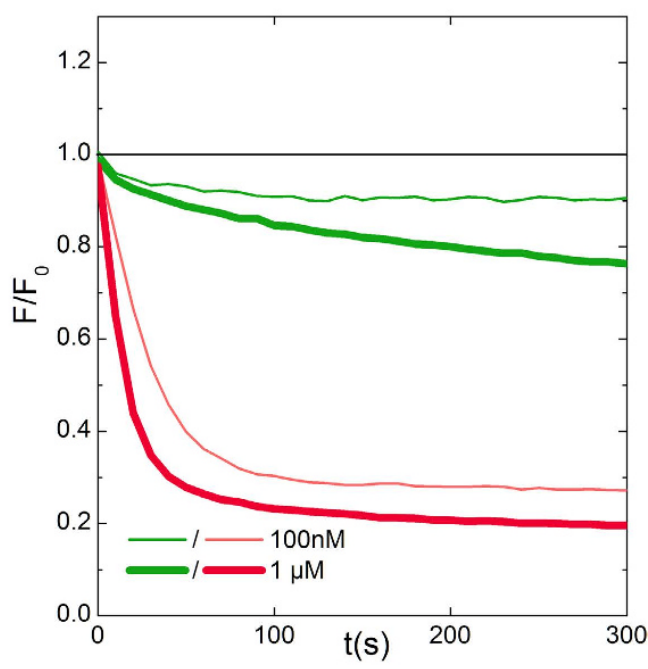

Figure 5. Gag self-assembly is sorting cholesterol but not sphingomyelin. (A and B) Typical fluorescence time course after addition of increasing FL-Gag concentrations on subsituted basic SLBs labeled with TFSPM (A) or TF-Chol (B). (C and D) Simultaneous fluorescence time course after addition of increasing concentrations of FL-Gag on substituted basic SLBs labelled with TF-SPM (in green) and BT-PIP 2 (in red) (C) or TF-Chol (in green) and BT-PIP 2 (in red) (D).

Altogether, these results show that HIV-1 Gag self-assembly is generating common $\mathrm{PIP}_{2}$ and cholesterol nanodomains while excluding sphingomyelin.

HIV-1 Gag is driving $\mathrm{PIP}_{2}$ and cholesterol nanoclustering independently of surrounding lipids. Because cellular PMs are complex lipid mixtures, we examined a possible role of this complexity on the lateral sorting of $\mathrm{PIP}_{2}$, Chol and SPM during HIV-1 Gag self-assembly. For that purpose, we used the two other different lipid mixture mimicking either the inner leaflet of cells PM ("inner-leaflet") or the "rafts" lipid mixture ("raft-mimicking") (see Table 1 and SI Table 1 for detailed lipid composition). We first compared the effect of FL-Gag self-assembly on $\mathrm{PIP}_{2}$ clustering for these three lipid compositions. No characteristic change in TF-PIP 2 fluorescence unquenching with increasing concentrations of FL-Gag (LUVs, Fig. 6A) or fluorescence quenching (SLBs, Fig. 6B) could be clearly detected amongst the three different lipid compositions. We then tested again Chol and SPM ability to partition into these FL-Gag induced PIP ${ }_{2}$ nanoclusters in the complex lipid mixtures. 
A

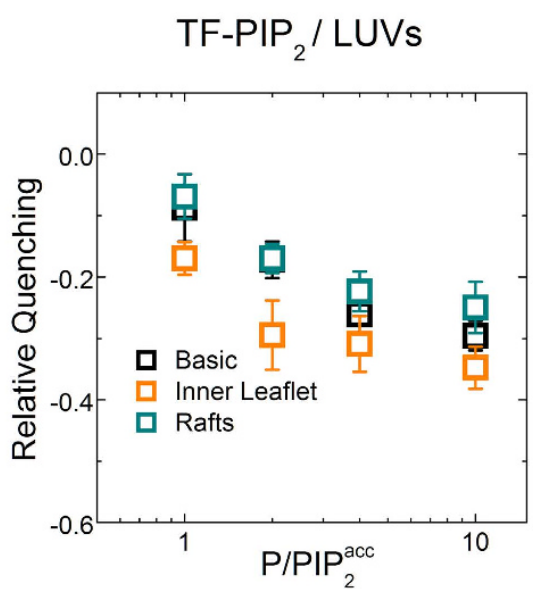

B $\mathrm{TF}^{\mathrm{PIP}}{ }_{2} / \mathrm{SLBS}$

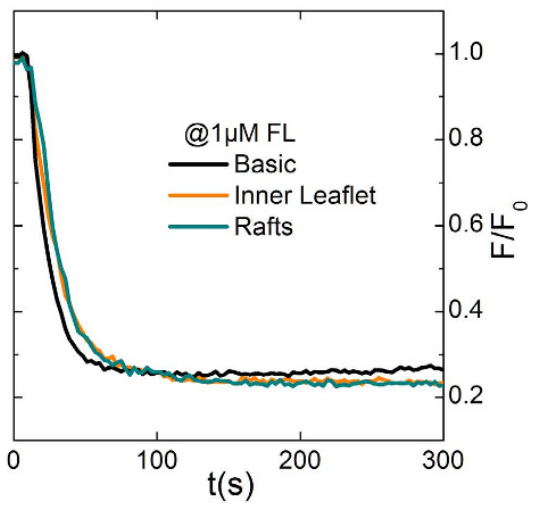

C

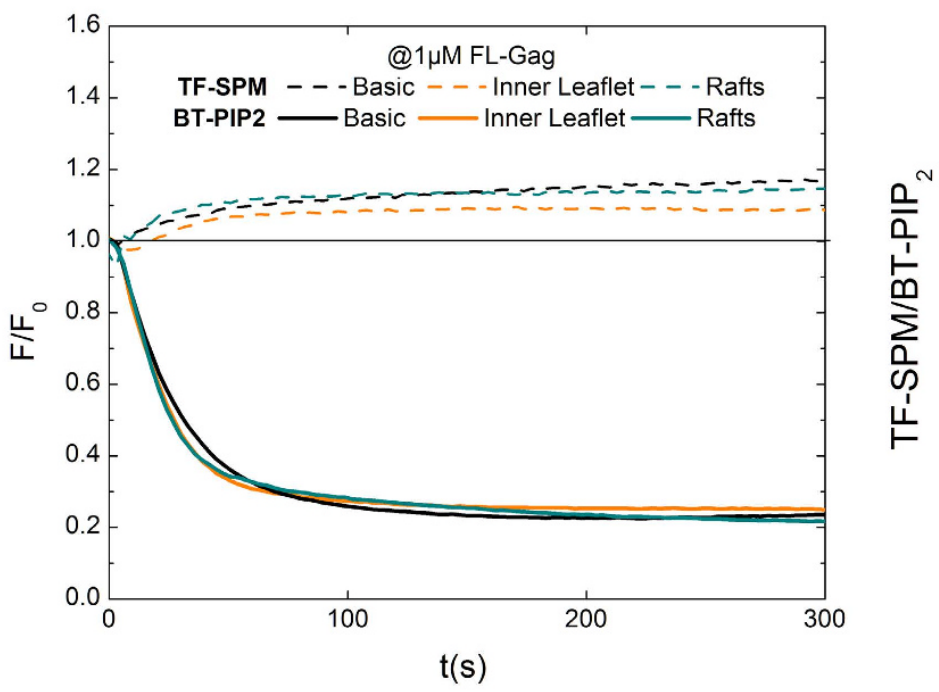

D

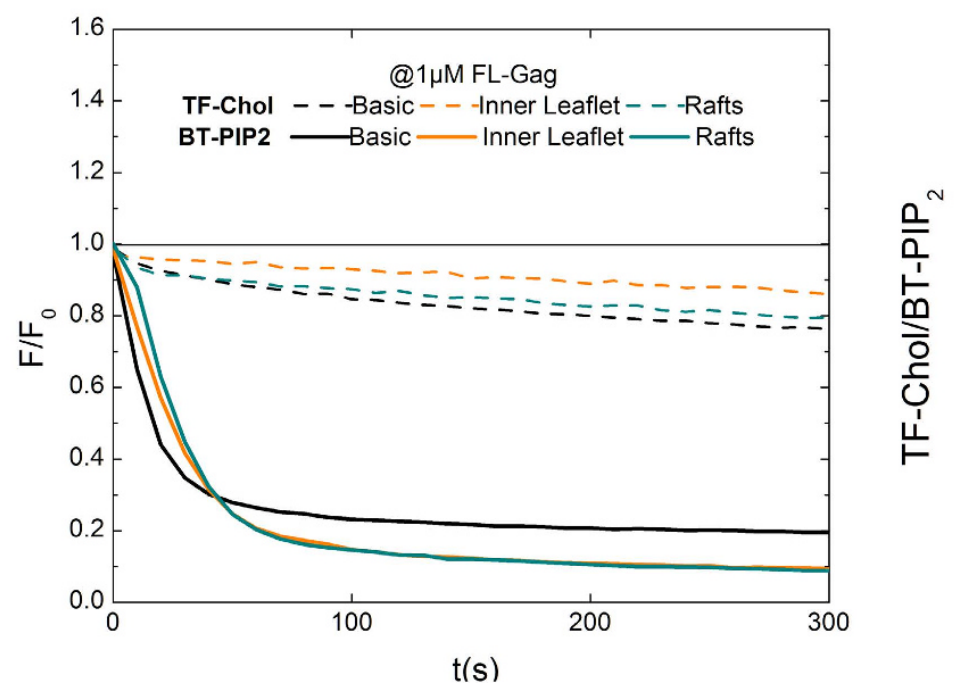

Figure 6. Gag $\mathrm{PIP}_{2}$ and Chol nanoclustering in complex membrane models. (A and $\left.\mathrm{B}\right) \mathrm{TF}-\mathrm{PIP}$ fluorescence changes in lipid membranes of different composition (basic, Inner Leaflet, Raft) using LUVs (A) relative quenching of at different $\mathrm{P} / \mathrm{PIP}_{2}^{a c c}$ ratio. mean \pm s.d., $n \geq 3$ ) or SLBs (B) typical time course of TF-PIP ${ }_{2}$ fluorescence after addition of $1 \mu \mathrm{M}$ FL-Gag.) (C) Simultaneous fluorescence time course obtained on "inner leaflet" SLBs for BT-PIP 2 (in red) and in green TF-SPM (left panel) or TF-Chol (right panel). (D) Simultaneous fluorescence time course obtained on "raft" SLBs for BT-PIP 2 (in red) and in green TF-SPM (left panel) or TFChol (right panel). 
Figure 6C (TF-SPM/BT-PIP $)$ and 6D $\left(\mathrm{TF}-\mathrm{Chol} / \mathrm{BT}-\mathrm{PIP}_{2}\right)$ exhibited the same tendency for the fluorescence time courses of labelled lipid upon FL-Gag addition on SLBs, independently of the lipid composition.

These results show that, as in the "basic" composition, in complex lipid mixtures the $\mathrm{PIP}_{2}$ nanoclusters induced by Gag self assembly are enriched in Chol but not in SPM. This clearly suggest that Gag is sorting these lipids during self-assembly independently of the surrounding lipids chemical nature and acts as the driving force for lipid reorganization during HIV-1 assembly.

\section{Discussion}

Although the MA domain is primary responsible for HIV-1 Gag binding to the PM, the ten times higher apparent affinity for membrane models observed here in the case of FL-Gag, P39 and WM confirms that the NC domains and CA-CA self-assembly of Gag are involved in membrane binding efficiency. Indeed, the NC domain of Gag alone has recently been described to bind to $\mathrm{PIP}_{2}$ containing lipid membranes with an apparent $K_{p}$ as high as $7 \mu \mathrm{M}^{33}$. It has also already been shown that the driving force for membrane association stems largely from ionic interactions between multimerized Gag and negatively charged phospholipids ${ }^{34}$.

Using micrometer scale phase separated GUVs, we have observed that Gag was mainly partitioning in $\mathrm{L}_{d}$ phases, i.e., more likely outside of lipid "rafts" (namely Chol and SPM enriched) domains. The lack of myristate in the different Gag variants tested here could explain the $\mathrm{Gag}_{d}$ phase localization ${ }^{35}$. Nevertheless, our results are consistent with the $\mathrm{L}_{d}$ phase GUV localization of multimerizable myr(+)MA protein observed by Keller et al. ${ }^{16}$.

However, it is known that cholesterol is crucial for virus infectivity ${ }^{36,37}$ and that Gag can sense cholesterol ${ }^{38,39}$. It was recently described that PIP $_{2}$ could form clusters in the presence of cholesterol alone ${ }^{40}$. Here we show that $\mathrm{PIP}_{2}$ and cholesterol are laterally redistributed upon Gag self-assembly on membranes and participate to the formation of cholesterol/PIP $/ \mathrm{P}_{2} \mathrm{Gag}$ enriched nanodomains. Importantly, we also show that sphingomyelin is not sorted during HIV-1 Gag self-assembly and is excluded from the Gag/PIP $2 /$ Chol nanoclusters. Taken together, these results suggest that binding and self-assembly of Gag protein does not occur in pre-existing lipid domains (such as "rafts") but that this self-assembly is more likely to induce lipid nanodomains.

It is known that plasma membrane lipid organization is not only driven by lipid-lipid interactions. For example, cytoplasmic proteins such as ezrin ${ }^{41,42}$, syntaxin $-1^{43}$ have also been described to induce or interact with PIP ${ }_{2}$ nanoclusters. As a general mechanism, proteins with basic interfaces can recruit acidic lipids that, in turn, can facilitate recruitment and clustering of these proteins into nanodomains ${ }^{44,45}$. A similar cooperative mechanism could also happen when HIV-1 Gag binds to PIP 2 during viral assembly at the plasma membrane. Interestingly, mesoscale organized structures such as cortical actin network has also been shown to play an important role in the lateral organization of not only transmembrane proteins, but inner leaflet plasma membrane lipids such as $\mathrm{PIP}_{2}{ }^{46}$ and more interestingly outer leaflet components such as GPi-anchored proteins ${ }^{47}$. Moreover, this cortical actin network has also been shown to play a role in HIV-1 Gag assembly at the PM of Jurkat T-cells ${ }^{48}$.

Since HIV-1 Gag has been often found in DRMs ${ }^{8}$, pre-existing outer leaflet "rafts" could be trapped by these nascent $\mathrm{PI}(4,5) \mathrm{P}_{2}$-Gag nanodomains through transbilayer coupling - as we already proposed ${ }^{18}$ or as it has been recently shown for lipid domains ${ }^{49}$ and for outer leaflet GPi anchored proteins ${ }^{50}$. Moreover, a recent study of the SPM dynamic in the cellular PM revealed that instead of clearly partitioning into nanodomains, SPM was mainly transiently trapped ${ }^{51}$. In the case of the ganglioside GM1, another lipid described as partitioning into $\mathrm{L}_{o}$ phases, its transient trapping was shown to depend on molecular pinning and interleaflet coupling between lipid tail domains ${ }^{52}$. This suggest that Gag could not only generates his own lipid nano-domains at the inner leaflet of the cellular PM but also induce the formation of domains in the outer leaflet of the cellular PM by transiently trapping SPM or other components.

Finally, we also observed that the matrix domain of Gag is not able to induce this $\mathrm{PIP}_{2}$ clustering, suggesting that, after maturation and particle release, the inner leaflet lipids of the virus envelope might be free to diffuse again.

\section{Conclusion}

We have shown using simple and cell mimicking lipid composition model membranes that Gag self-assembly is inducing nano-clusters enriched in $\mathrm{PIP}_{2}$ and cholesterol, instead of partitioning to pre-existing ones. This lipid nano-clustering does not require sphingomyelin and mainly occurs out of the $\mathrm{L}_{o}$ phases in GUVs. Further, the different lipid composition tested here does not strikingly affect the capacity of Gag to induce these lipid nano-clusters suggesting that Gag is able to sort its own lipids independently of the surrounding lipids.

\section{Methods}

All experiments were performed at room temperature (RT). Experimental buffer was Hepes $10 \mathrm{mM}, \mathrm{pH}=7.4$, $\mathrm{KCl} 150 \mathrm{mM}$, EDTA $2 \mathrm{mM}$ except for QCM-D binding experiments. All images were acquired with a Zeiss LSM 780 microscope (Carl Zeiss, Inc.) using a 63x NA 1.4 oil objective and quantified using Image J software (NIH, MD, USA). Detailed materials, methods for model membranes (LUV, GUV, SLB) preparation and for protein purification are in SI.

Binding experiments. $\quad \mathrm{K}_{p}$ were determined on basic lipid composition (EPC 68\%, BPS 30\% \& PI $(4,5) \mathrm{P}_{2} 2 \%$ for LUVs or POPC $68 \%$, POPS $30 \%$ \& PI $(4,5) \mathrm{P}_{2} 2 \%$ for SLBs). Methods used were either cosedimentation assays for LUVs or QCM-D experiments on SLBs. Co-sedimentation assays were made at $1 \mu \mathrm{M}$ protein concentration with varying concentrations of total accessible lipids from 0.07 to $2250 \mu \mathrm{M}$ in $100 \mu l$ of the same buffer that the one used in quenching experiments, according to the protocol in ref. 53. After $15 \mathrm{~min}$ of incubation at room temperature, samples were centrifuged at $220,000 \mathrm{~g}$ for $1 \mathrm{~h}$ at $4^{\circ} \mathrm{C}$ using a Beckman Coulter's TLA 100 rotor. The top $80 \mu l$ was considered as supeRNAtant (S) and the remaining $20 \mu l$ diluted with $60 \mu l$ of working buffer as pellet (P). Pellet and supeRNAtant were analyzed on a 10\% SDS-PAGE and stained using coomassie blue. Quantifications 
were made using Image J software (National Institutes of Health, MD, USA). SLBs were prepared with $0.1 \mathrm{mg} \cdot \mathrm{mL}^{-1}$ liposomes flowing at $10 \mu \mathrm{L} \cdot \mathrm{min}^{-1}$ for 10-20 min on a UV-treated $\mathrm{SiO}_{2}$ surface of Q-sensor fixed in a Q-Sense Flow module, QFM 401 Biolin Scientific, Sweden). Stable SLBs were rinsed with citrate buffer (NaCitrate $10 \mathrm{mM}$, $100 \mathrm{mM} \mathrm{NaCl}$, and $0.5 \mathrm{mM}$ EGTA, pH 4.6) and then with injection buffer (5 mM Tris \& $100 \mathrm{mM} \mathrm{NaCl} \mathrm{pH} \mathrm{7.4).}$ At equilibrium, $200 \mu \mathrm{L}$ of increasing protein concentration was successively injected into the flow chamber followed by rinsing steps in between. The same was repeated with increasing protein concentrations until saturation. Sensorgrams were normalized to third harmonic in the case of varying harmonic curves. $\Delta F$ (plateau values) were used to measure the lipid SLB surface fraction of protein bound. The fraction of protein bound is related to an apparent association constant $K$ (i.e, the reciprocal of the apparent partitioning constant, $K_{p}$ ) following the equation:

$$
\frac{[P L]}{[P L]_{\max }}=\frac{K[L]_{a c c}}{1+K[L]_{a c c}}
$$

where the percentage of protein bound is $[P L]=I_{\text {Pellet }} /\left(I_{P}+I_{S}\right)$ in the case of the co-sedimentation experiments ( $I_{P} \& I_{S}$ experimental intensities of pellet and supeRNAtant and $I_{P e l l e t}=I_{P}-0.2 . I_{S}$, for dilution compensation). In the case of QCM-d experiments, apparent $K_{p}$ values were obtained using Eq. 1 formulated as a function of $[P]$ instead of $[L]$.

Image analysis of GUVs. GUVs were deposited on coverslips coated with casein $\left(2 \mathrm{mg} \cdot \mathrm{mL}^{-1}\right)$ fitted in an Attofluor cell chamber (Thermo Fisher Scientific, Inc.). Labeled FL-Gag was added to the buffer. Spectral images were acquired at constant excitation intensity at $488 \mathrm{~nm}, 561 \mathrm{~nm}$ and $633 \mathrm{~nm}$ using an emission spectral range between 499 and $690 \mathrm{~nm}$ with a $8 \mathrm{~nm}$ resolution. Linear unmixing of these images was done to avoid potential bleed-through due to fluorophore emission overlapping. To establish the spatial auto-correlation of the fluorescence intensity of TF-PIP 2 or A594-FL-Gag on basic composition GUVs, the change in intensity was plotted along the GUV using the following integration:

$$
I(r, \theta)=\frac{1}{N} \sum_{d r=r-2 \Delta r}^{d r=r+2 \Delta r} \sum_{\theta=\theta}^{\theta=\theta+\Delta \theta} I_{N}(r+d r, \theta+\Delta \theta)
$$

Intensity was plotted with $r \cdot \sin \theta$ as the length unit. The obtained curves were autocorrelated using either the autocorr or the xcorr function of Matlab R2015 (Mathworks ${ }^{\circledR}$ ).

Intensity partition of each label in the case of raft GUVs was determined using the following equations:

$$
I_{\text {in }}=\sum_{\theta=\theta_{i}}^{\theta=\theta_{o}} I\left(r_{G U V}, \theta\right) \Delta \theta / \sum_{\theta=0}^{\theta=2 \pi} I\left(r_{G U V}, \theta\right) \Delta \theta, I_{\text {out }}=1-I_{\text {in }}
$$

$\theta_{i}$ and $\theta_{o}$ were determined with the help of Alexa647-CtxB intensity circular profile.

Fluorescence quenching measurements. For LUV experiments, fluorescence was monitored using a spectrometer (Photon Technologies InteRNAtional, Inc.) with $\lambda_{e x c}=485 \pm 2 \mathrm{~nm}$ and $\lambda_{e m}=520 \pm 10 \mathrm{~nm}$. Excitation lamp intensity was calibrated using the Raman spectrum of pure water and its fluctuations corrected every second. For every interaction assay on LUVs, the obtained intensity curve was corrected for both bleaching and dilution effect and then normalized to the mean intensity before injection. In the case of SLBs, fluorescence was monitored by acquiring images using the Zeiss definite focus system to avoid any z-drift with a 2-photons excitation $(930 \mathrm{~nm}$ ) every $5 \mathrm{~s}$ (in order to strongly reduce photobleaching). TF-lipids fluorescence was acquired at $520 \pm 30 \mathrm{~nm}$ and BT-PIP 2 at $600 \pm 40$. Mean intensity of each image was normalized to the intensity before injection.

Self-assembly assay on LUVs. $\quad 100 \mu \mathrm{L}$ of the basic LUVs was incubated at room temperature for $15 \mathrm{~min}$ with either $0.9 \mu \mathrm{M}$ or $4.5 \mu \mathrm{M}$ of FL-Gag or WM. After centrifugation at $10,000 \mathrm{~g}$ for $5 \mathrm{~min}$ at $4 \mathrm{C}, 20 \mu \mathrm{L}$ of the supeRNAtant was loaded on a 10\% native-PAGE and proceed for immunoblot as in ref. 53. FL-Gag and WM were detected by a primary anti-capsid antibody (HIV-1 p24 NIH AIDS Reagents) followed by secondary antibody goat anti-mouse HRP conjugated. Membrane was revealed by Femto substrate (Thermo-scientific) and imaged by a G:Box (Syngene).

Fluorescence Recovery After Photobleaching. The image sequence was acquired at $20 \mathrm{~Hz}$ using the $488 \mathrm{~nm}$ line of an $\mathrm{Ar}^{+}$laser at a very low power to avoid photobleaching. After $2.5 \mathrm{~s}, 3$ regions of interests (ROI), of $1 \mu \mathrm{m}$ radius each, were rapidly photobleached $(\mathrm{t}<60 \mathrm{~ms})$ at maximal laser power. Fluorescence recovery was monitored for $15 \mathrm{~s}$. The recovery curves were obtained as in ref. 54 . In order to correctly estimate $F_{0}$ (the fluorescence intensity immediately after the end of the bleach) and $F_{15 s}$, the curves were fitted as in ref. 54 . The normalized fractional recovery $(N F R)$, is defined as:

$$
N F R=\frac{F_{15 s}-F_{0}}{1-F_{0}}
$$




\section{References}

1. Balasubramaniam, M. \& Freed, E. O. New insights into HIV assembly and trafficking. Physiology (Bethesda) 26, 236-251 URL http:// dx.doi.org/10.1152/physiol.00051.2010 (2011).

2. Bieniasz, P. D. The cell biology of HIV-1 virion genesis. Cell Host Microbe 5, 550-558 URL http://dx.doi.org/10.1016/j. chom.2009.05.015 (2009)

3. Muriaux, D. \& Darlix, J.-L. Properties and functions of the nucleocapsid protein in virus assembly. RNA Biol 7, 744-753 (2010).

4. Hendrix, J. et al. Live-cell observation of cytosolic HIV-1 assembly onset reveals RNA-interacting Gag oligomers. J Cell Biol 210, 629-646 URL http://dx.doi.org/10.1083/jcb.201504006 (2015).

5. Saad, J. S. et al. Structural basis for targeting HIV-1 Gag proteins to the plasma membrane for virus assembly. Proc Natl Acad Sci USA 103, 11364-11369 URL http://dx.doi.org/10.1073/pnas.0602818103 (2006).

6. Ono, A., Ablan, S. D., Lockett, S. J., Nagashima, K. \& Freed, E. O. Phosphatidylinositol $(4,5)$ bisphosphate regulates HIV-1 Gag targeting to the plasma membrane. Proc Natl Acad Sci USA 101, 14889-14894 URL http://dx.doi.org/10.1073/pnas.0405596101 (2004).

7. Chan, R. et al. Retroviruses human immunodeficiency virus and murine leukemia virus are enriched in phosphoinositides. $J$ Virol 82, 11228-11238 URL http://dx.doi.org/10.1128/JVI.00981-08 (2008).

8. Ono, A. \& Freed, E. O. Plasma membrane rafts play a critical role in HIV-1 assembly and release. Proc Natl Acad Sci USA 98, 13925-13930 URL http://dx.doi.org/10.1073/pnas.241320298 (2001).

9. Callahan, E. M. \& Wills, J. W. Repositioning basic residues in the M domain of the rous sarcoma virus Gag protein. $J$ Virol 74, 11222-11229 (2000).

10. Holm, K., Weclewicz, K., Hewson, R. \& Suomalainen, M. Human immunodeficiency virus type 1 assembly and lipid rafts: Pr55(Gag) associates with membrane domains that are largely resistant to brij98 but sensitive to triton X-100. J Virol 77, 4805-4817 (2003).

11. Campbell, S. et al. The raft-promoting property of virion-associated cholesterol, but not the presence of virion-associated brij 98 rafts, is a determinant of human immunodeficiency virus type 1 infectivity. J Virol 78, 10556-10565 URL http://dx.doi.org/10.1128/ JVI.78.19.10556-10565.2004 (2004).

12. Pike, L. J. Rafts defined: a report on the keystone symposium on lipid rafts and cell function. J Lipid Res 47, 1597-1598 URL http:// dx.doi.org/10.1194/jlr.E600002-JLR200 (2006).

13. Sengupta, P., Hammond, A., Holowka, D. \& Baird, B. Structural determinants for partitioning of lipids and proteins between coexisting fluid phases in giant plasma membrane vesicles. Biochimica et Biophysica Acta (BBA) - Biomembranes 1778, 20-32 URL http://www.sciencedirect.com/science/article/pii/S0005273607003240 (2008).

14. Charlier, L. et al. Coarse-grained simulations of the HIV-1 matrix protein anchoring: revisiting its assembly on membrane domains. Biophys J 106, 577-585 URL http://dx.doi.org/10.1016/j.bpj.2013.12.019 (2014).

15. Mercredi, P. Y. et al. Structural and molecular determinants of membrane binding by the HIV-1 matrix protein. J Mol Biol URL http://dx.doi.org/10.1016/j.jmb.2016.03.005 (2016).

16. Keller, H., Kräusslich, H.-G. \& Schwille, P. Multimerizable HIV Gag derivative binds to the liquid-disordered phase in model membranes. Cell Microbiol 15, 237-247 URL http://dx.doi.org/10.1111/cmi.12064 (2013).

17. Wen, Y., Dick, R., Feigenson, G. \& Vogt, V. Effects of membrane charge and order on membrane binding of the retroviral structural protein gag. J Virol URL http://dx.doi.org/10.1128/JVI.01102-16 (2016).

18. Kerviel, A., Thomas, A., Chaloin, L., Favard, C. \& Muriaux, D. Virus assembly and plasma membrane domains: which came first? Virus Res 171, 332-340 URL http://dx.doi.org/10.1016/j.virusres.2012.08.014 (2013).

19. Wawrezinieck, L., Rigneault, H., Marguet, D. \& Lenne, P.-F. Fluorescence correlation spectroscopy diffusion laws to probe the submicron cell membrane organization. Biophys J 89, 4029-4042 URL http://dx.doi.org/10.1529/biophysj.105.067959 (2005).

20. Favard, C., Wenger, J., Lenne, P.-F. \& Rigneault, H. FCS diffusion laws in two-phase lipid membranes: determination of domain mean size by experiments and Monte Carlo simulations. Biophys J 100, 1242-1251 URL http://dx.doi.org/10.1016/j.bpj.2010.12.3738 (2011).

21. Eggeling, C. et al. Direct observation of the nanoscale dynamics of membrane lipids in a living cell. Nature 457, 1159-1162 URL http://dx.doi.org/10.1038/nature07596 (2009)

22. Andrade, D. M. et al. Cortical actin networks induce spatio-temporal confinement of phospholipids in the plasma membrane-a minimally invasive investigation by STED-FCS. Sci Rep 5, 11454 URL http://dx.doi.org/10.1038/srep11454 (2015).

23. Wu, H.-M., Lin, Y.-H., Yen, T.-C. \& Hsieh, C.-L. Nanoscopic substructures of raft-mimetic liquid-ordered membrane domains revealed by high-speed single-particle tracking. Sci Rep 6, 20542 URL http://dx.doi.org/10.1038/srep20542 (2016).

24. Gambhir, A. et al. Electrostatic sequestration of PIP2 on phospholipid membranes by basic/aromatic regions of proteins. Biophys J 86, 2188-2207 URL http://dx.doi.org/10.1016/S0006-3495(04)74278-2 (2004).

25. Puff, N., Watanabe, C., Seigneuret, M., Angelova, M. I. \& Staneva, G. Lo/Ld phase coexistence modulation induced by GM1. Biochim Biophys Acta 1838, 2105-2114 URL http://dx.doi.org/10.1016/j.bbamem.2014.05.002 (2014).

26. Heberle, F. A., Wu, J., Goh, S. L., Petruzielo, R. S. \& Feigenson, G. W. Comparison of three teRNAry lipid bilayer mixtures: FRET and ESR reveal nanodomains. Biophys J 99, 3309-3318 URL http://dx.doi.org/10.1016/j.bpj.2010.09.064 (2010).

27. Chukkapalli, V., Hogue, I. B., Boyko, V., Hu, W.-S. \& Ono, A. Interaction between the human immunodeficiency virus type 1 Gag matrix domain and phosphatidylinositol-(4,5)-bisphosphate is essential for efficient Gag membrane binding. J Virol 82, 2405-2417 URL http://dx.doi.org/10.1128/JVI.01614-07 (2008).

28. Ingólfsson, H. I. et al. Lipid organization of the plasma membrane. J Am Chem Soc 136, 14554-14559 URL http://dx.doi. org/10.1021/ja507832e (2014).

29. Datta, S. A. K. et al. Conformation of the HIV-1 Gag protein in solution. J Mol Biol 365, 812-824 URL http://dx.doi.org/10.1016/j. jmb.2006.10.073. (2007).

30. Macia, E. et al. The pleckstrin homology domain of the Arf6-specific exchange factor EFA6 localizes to the plasma membrane by interacting with phosphatidylinositol 4,5-bisphosphate and F-actin. J Biol Chem 283, 19836-19844 URL http://dx.doi.org/10.1074/ jbc.M800781200 (2008)

31. Momany, C. et al. Crystal structure of dimeric HIV-1 capsid protein. Nat Struct Biol 3, 763-770 (1996)

32. Fung, B. K. \& Stryer, L. Surface density determination in membranes by fluorescence energy transfer. Biochemistry 17, 5241-5248 (1978).

33. Kempf, N. et al. The HIV-1 nucleocapsid protein recruits negatively charged lipids to ensure its optimal binding to lipid membranes. J Virol 89, 1756-1767 URL http://dx.doi.org/10.1128/JVI.02931-14 (2015).

34. Dalton, A. K., Ako-Adjei, D., Murray, P. S., Murray, D. \& Vogt, V. M. Electrostatic interactions drive membrane association of the human immunodeficiency virus type 1 Gag MA domain. J Virol 81, 6434-6445 URL http://dx.doi.org/10.1128/JVI.02757-06 (2007).

35. Lindwasser, O. W. \& Resh, M. D. Myristoylation as a target for inhibiting HIV assembly: unsaturated fatty acids block viral budding. Proc Natl Acad Sci USA 99, 13037-13042 URL http://dx.doi.org/10.1073/pnas.212409999 (2002).

36. Hawkes, D. et al. Properties of HIV-1 associated cholesterol in addition to raft formation are important for virus infection. Virus Res 210, 18-21 URL http://dx.doi.org/10.1016/j.virusres.2015.06.023 (2015).

37. Campbell, S. M., Crowe, S. M. \& Mak, J. Virion-associated cholesterol is critical for the maintenance of HIV-1 structure and infectivity. AIDS 16, 2253-2261 (2002).

38. Dick, R. A., Goh, S. L., Feigenson, G. W. \& Vogt, V. M. HIV-1 Gag protein can sense the cholesterol and acyl chain environment in model membranes. Proc Natl Acad Sci USA 109, 18761-18766 URL http://dx.doi.org/10.1073/pnas.1209408109 (2012). 
39. Barros, M. et al. Membrane binding of HIV-1 matrix protein: Dependence on bilayer composition and protein lipidation. J Virol 90, 4544-4555 URL http://dx.doi.org/10.1128/JVI.02820-15 (2016).

40. Jiang, Z., Redfern, R. E., Isler, Y., Ross, A. H. \& Gericke, A. Cholesterol stabilizes fluid phosphoinositide domains. Chem Phys Lipids 182, 52-61 URL http://dx.doi.org/10.1016/j.chemphyslip.2014.02.003 (2014).

41. Chen, X. et al. Phosphatidylinositol 4,5-bisphosphate clusters the cell adhesion molecule CD44 and assembles a specific CD44-Ezrin heterocomplex, as revealed by small angle neutron scattering. J Biol Chem 290, 6639-6652 URL http://dx.doi.org/10.1074/jbc. M114.589523 (2015).

42. Al-Momany, A., Li, L., Alexander, R. T. \& Ballermann, B. J. Clustered PI(4,5)P2 accumulation and Ezrin phosphorylation in response to Clic5a. J Cell Sci 127, 5164-5178 URL http://dx.doi.org/10.1242/jcs.147744 (2014).

43. Honigmann, A. et al. Phosphatidylinositol 4,5-bisphosphate clusters act as molecular beacons for vesicle recruitment. Nat Struct Mol Biol 20, 679-686 URL http://dx.doi.org/10.1038/nsmb.2570. (2013).

44. van den Bogaart, G. et al. Membrane protein sequestering by ionic protein-lipid interactions. Nature 479, 552-555 URL http:// dx.doi.org/10.1038/nature10545 (2011).

45. McLaughlin, S. \& Murray, D. Plasma membrane phosphoinositide organization by protein electrostatics. Nature 438, 605-611 URL http://dx.doi.org/10.1038/nature04398 (2005).

46. Golebiewska, U. et al. Evidence for a fence that impedes the diffusion of phosphatidylinositol 4,5-bisphosphate out of the forming phagosomes of macrophages. Mol Biol Cell 22, 3498-3507 URL http://dx.doi.org/10.1091/mbc.E11-02-0114 (2011).

47. Goswami, D. et al. Nanoclusters of GPi-anchored proteins are formed by cortical actin-driven activity. Cell 135, 1085-1097 URL http://dx.doi.org/10.1016/j.cell.2008.11.032 (2008).

48. Thomas, A. et al. Involvement of the Rac1-IRSp53-Wave2-Arp2/3 signaling pathway in HIV-1 Gag particle release in CD4 T cells. J Virol 89, 8162-8181 URL http://dx.doi.org/10.1128/JVI.00469-15 (2015).

49. Blosser, M. C., Honerkamp-Smith, A. R., Han, T., Haataja, M. \& Keller, S. Transbilayer colocalization of lipid domains explained via measurement of strong coupling parameters. Biophysical JouRNAl 109, 2317-2327 URL http://www.sciencedirect.com/science/ article/pii/S0006349515011066 (2015).

50. Raghupathy, R. et al. Transbilayer lipid interactions mediate nanoclustering of lipid-anchored proteins. Cell 161, 581-594 URL http://dx.doi.org/10.1016/j.cell.2015.03.048 (2015).

51. Honigmann, A. et al. Scanning STED-FCS reveals spatiotemporal heterogeneity of lipid interaction in the plasma membrane of living cells. Nat Commun 5, 5412, URL http://dx.doi.org/10.1038/ncomms6412 (2014).

52. Spillane, K. M. et al. High-speed single-particle tracking of GM1 in model membranes reveals anomalous diffusion due to interleaflet coupling and molecular pinning. Nano Lett 14, 5390-5397, URL http://dx.doi.org/10.1021/nl502536u (2014).

53. Hamard-Peron, E. et al. Targeting of murine leukemia virus Gag to the plasma membrane is mediated by PI(4,5)P2/PS and a polybasic region in the matrix. J Virol 84, 503-515, URL http://dx.doi.org/10.1128/JVI.01134-09 (2010).

54. Escoffre, J. M., Hubert, M., Teissié, J., Rols, M. P. \& Favard, C. Evidence for electro-induced membrane defects assessed by lateral mobility measurement of a GPi anchored protein. Eur Biophys J 43, 277-286, URL http://dx.doi.org/10.1007/s00249-014-0961-1 (2014).

55. Ehrlich, L. S., Fong, S., Scarlata, S., Zybarth, G. \& Carter, C. Partitioning of HIV-1 Gag and Gag-related proteins to membranes. Biochemistry 35, 3933-3943, URL http://dx.doi.org/10.1021/bi952337x (1996).

56. Wang, J. et al. Lateral sequestration of phosphatidylinositol 4,5-bisphosphate by the basic effector domain of myristoylated alaninerich c kinase substrate is due to nonspecific electrostatic interactions. J Biol Chem 277, 34401-34412, URL http://dx.doi.org/10.1074/ jbc.M203954200 (2002)

\section{Acknowledgements}

We are thankful to Dr. Olivier Coux for his help with protein purification and Dr. Michel Franco for the gift of PH-EFA6 plasmid. We thank the Montpellier RIO Imaging (MRI) microscopy facilities. This work has been funded by ANR (ANR-13-BSV5-0006-01). C.P. acknowledges the European Commission (FP7) for a grant from the European Research Council (GA259370). N.Y. is a recipient of the European Community Erasmus Mundus $\mathrm{PhD}$ fellowship. C.F. and D.M. are part of the CNRS GDR MIV consortium.

\section{Author Contributions}

D.M. and C.F. developed the concept of the study. N.Y., Q.L., H.T., C.F. conducted the experiments. N.Y., Q.L. and D.M. and C.F. conducted data interpretation. C.F., N.Y. and D.M. drafted the manuscript. J.M., C.P. contributed to data interpretation and manuscript drafting.

\section{Additional Information}

Supplementary information accompanies this paper at http://www.nature.com/srep

Competing financial interests: The authors declare no competing financial interests.

How to cite this article: Yandrapalli, N. et al. Self assembly of HIV-1 Gag protein on lipid membranes generates $\mathrm{PI}(4,5) \mathrm{P}_{2} /$ Cholesterol nanoclusters. Sci. Rep. 6, 39332; doi: 10.1038/srep39332 (2016).

Publisher's note: Springer Nature remains neutral with regard to jurisdictional claims in published maps and institutional affiliations.

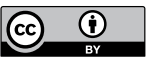

This work is licensed under a Creative Commons Attribution 4.0 International License. The images or other third party material in this article are included in the article's Creative Commons license, unless indicated otherwise in the credit line; if the material is not included under the Creative Commons license, users will need to obtain permission from the license holder to reproduce the material. To view a copy of this license, visit http://creativecommons.org/licenses/by/4.0/

(C) The Author(s) 2016 